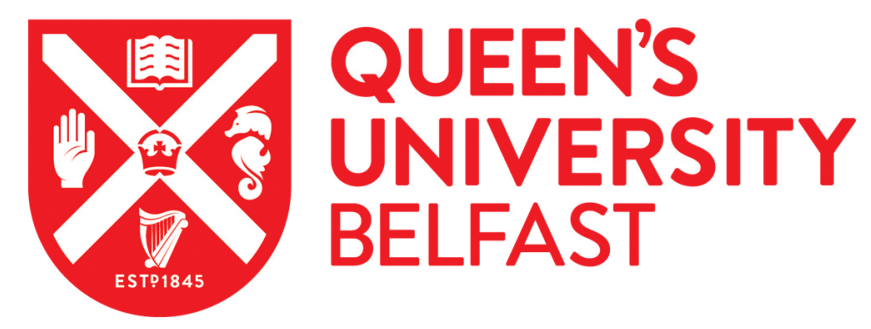

\title{
Assessment of the effect of Cellets' particle size on the flow in a Wurster fluid-bed coater via powder rheology
}

Mohylyuk, V., Styliari, I. D., Novykov, D., Pikett, R., \& Dattani, R. (2019). Assessment of the effect of Cellets' particle size on the flow in a Wurster fluid-bed coater via powder rheology. Journal of Drug Delivery Science and Technology, 54, [101320]. https://doi.org/10.1016/j.jddst.2019.101320

Published in:

Journal of Drug Delivery Science and Technology

Document Version:

Peer reviewed version

Queen's University Belfast - Research Portal:

Link to publication record in Queen's University Belfast Research Portal

Publisher rights

Copyright 2019 Elsevier.

This manuscript is distributed under a Creative Commons Attribution-NonCommercial-NoDerivs License

(https://creativecommons.org/licenses/by-nc-nd/4.0/), which permits distribution and reproduction for non-commercial purposes, provided the author and source are cited.

\section{General rights}

Copyright for the publications made accessible via the Queen's University Belfast Research Portal is retained by the author(s) and / or other copyright owners and it is a condition of accessing these publications that users recognise and abide by the legal requirements associated with these rights.

Take down policy

The Research Portal is Queen's institutional repository that provides access to Queen's research output. Every effort has been made to ensure that content in the Research Portal does not infringe any person's rights, or applicable UK laws. If you discover content in the Research Portal that you believe breaches copyright or violates any law, please contact openaccess@qub.ac.uk. 


\section{Journal Pre-proof}

Assessment of the effect of Cellets' particle size on the flow in a Wurster fluid-bed coater via powder rheology

Mohylyuk Valentyn, Styliari loanna Danai, Novykov Dmytryi, Pikett Reiss, Dattani Rajeev

PII:

$$
\text { S1773-2247(19)31198-0 }
$$

DOI: https://doi.org/10.1016/j.jddst.2019.101320

Reference: JDDST 101320

To appear in: Journal of Drug Delivery Science and Technology

Received Date: 13 August 2019

Accepted Date: 8 October 2019

Please cite this article as: M. Valentyn, S.I. Danai, N. Dmytryi, P. Reiss, D. Rajeev, Assessment of the effect of Cellets' particle size on the flow in a Wurster fluid-bed coater via powder rheology, Journal of Drug Delivery Science and Technology (2019), doi: https://doi.org/10.1016/j.jddst.2019.101320.

This is a PDF file of an article that has undergone enhancements after acceptance, such as the addition of a cover page and metadata, and formatting for readability, but it is not yet the definitive version of record. This version will undergo additional copyediting, typesetting and review before it is published in its final form, but we are providing this version to give early visibility of the article. Please note that, during the production process, errors may be discovered which could affect the content, and all legal disclaimers that apply to the journal pertain.

(C) 2019 Published by Elsevier B.V. 
4 Mohylyuk Valentyn ${ }^{1,2,3, *}$, Styliari Ioanna Danai ${ }^{2}$, Novykov Dmytryi ${ }^{3}$, Pikett Reiss ${ }^{4}$,

5 Dattani Rajeev ${ }^{4}$

6

7

Graphical abstract

\section{fluid-bed coater via powder rheology \\ Assessment of the effect of Cellets' particle size on the flow in a Wurster}

\footnotetext{
${ }^{1}$ Pharmaceutical Engineering Group, School of Pharmacy, Queen's University Belfast, 97 Lisburn
} Road, Belfast, BT9 7BL, UK

${ }^{2}$ Department of Clinical and Pharmaceutical Sciences, University of Hertfordshire, College Ln., Hatfield, AL10 9AB, UK

${ }^{3}$ Department of Biomedical Technology, Ukraine University, 23 Lvivska Street, Kyiv, 03115, Ukraine

${ }^{4}$ Freeman Technology, Tewkesbury Business Park, Severn Drive, Tewkesbury, GL20 8D, UK

* Contact author: Dr Valentyn Mohylyuk

Email: v.mohylyuk@qub.ac.uk

Tel: +447410807295

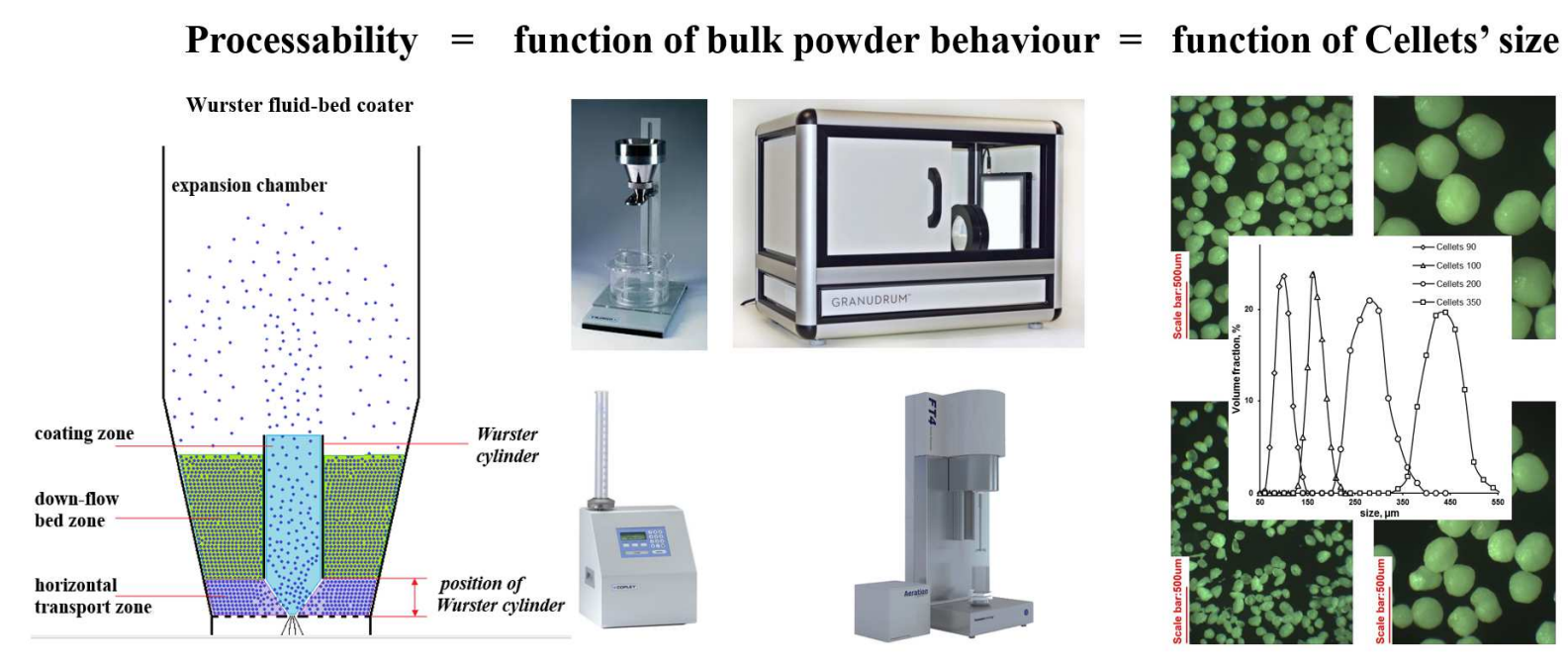




\section{Abstract}

23 The main objective of this study was to investigate the effect of microcrystalline cellulose 24 (MCC) spheres (Cellets) size effect on the powder properties to assess the possible impact of 25 the Cellets' size on the powder behaviour in the down-flow bed and horizontal transport zone 26 when a Wurster fluid-bed coater is used.

27 The particle size distribution of Cellets was determined using optical digital microscopy. 28 Standard pharmacopoeia methods as bulk/tapped density and flow rate measured with 29 gravitational funnel method as well as investigation of dynamic angle of repose and dynamic 30 cohesivity index with a rotating drum tester (GranuDrum) and conditioned bulk density, basic 31 flowability energy, specific energy, pressure drop, permeability and compressibility by 32 powder rheometer (FT4 Powder Rheometer ${ }^{\circledR}$ ), were employed to characterise the powder's properties of Cellets 90, 100, 200 and 350 ( $\mathrm{D}_{50}$-size from 94 to $\left.424 \mu \mathrm{m}\right)$ alone or premixed with $0.5 \% \mathrm{w} / \mathrm{w}$ magnesium stearate.

Specific powder rheology methods were proposed for characterisation of Cellets' behaviour 36 in down-flow bed, the horizontal transport and coating zone.

37 The level of Cellets' processability decreasing in the Wurster fluid-bed coater with decreasing of Cellets size $\left(D_{50}\right)$ from 425 to $94 \mu \mathrm{m}$ was established with different powder rheology methods.

41 Key words: Cellets; pellets; powder rheology; flowability; fluid-bed; coating; processability 


\section{Introduction}

Microcrystalline cellulose (MCC) is one of the most widely used excipients in the formulation of solid dosage forms. Spherical MCC particles, such as Cellets are becoming increasingly popular as the inert core in the fluid-bed coating process [1,2]. Coated pellets' particle size can influence the solid dosage form's sedimentation rate [3], uniformity [4] and mouthfeel [5]. Depending on the specific final product, Cellets with different particle sizes could be used as inert cores in the multi-layered pellets [1].

A fluid-bed coater with a Wurster cylinder is one of the most commonly used operations for pellet coating and is a circulating process, but it doesn't contain a fluid-bed in the traditional sense [6]. A Wurster cylinder-containing (two-compartment) design of processing column was intended to reduce inter-particle weight gain variability (compared to a one-compartment design) [7].

A few different zones are identified in fluid-bed coater with a Wurster cylinder: the coating zone, the expansion chamber, the down-flow bed zone, and the horizontal transport zone (Figure 1). Cores come into the coating zone via a high velocity air flow, rising and then additionally achieving atomisation air flow and droplets are sprayed from a nozzle. After passing through the Wurster cylinder, cores settle in the expansion chamber on the surface of the down-flow bed and gradually move downwards. Between the bottom part of down-flow bed zone and coating zone is the horizontal transport zone. The adjustable position of the Wurster cylinder (the distance between the air distribution disk and the bottom edge of the Wurster cylinder) controls powder flow rate per unit area. The horizontal transport of powder could be considered similar to pneumatic conveying. The fluidised-bed region is only present in the horizontal transport zone and reduces the friction between particles, which helps to convey them to the coating zone. $[6,8]$

The particle size, density, surface area, smoothness of the surface and particle-to-particle interaction are amongst the most important powder properties which affects the process performance in different zones. The ideal process settings should provide enough powder flow in the down-flow bed zone and horizontal transport zone to ensure that within the coating zone all droplets will coat the powder particles. Additionally, air flow and atomisation air flow should provide a reasonable throughput of particles through the Wurster cylinder [9]. 
Recently, a new technological platform has been developed overcoming the particle size limitations during sustained release microparticle coating with an aqueous polymer dispersion using Wurster fluid bed coater. Periodically adding a small quantity of dry powder glidant during coating overcame this issue and substantially improved product yield due to the improvement of the powder's flow [10].

The purpose of current work is to investigate the effect of Cellets' particle size on powder properties and their possible effect on the processing in a fluid-bed coater with a Wurster cylinder. The behaviour in the down-flow bed and horizontal transport zone is a point of interest. As an additional task, to assess the effect of magnesium stearate at a concentration level of $0.5 \% \mathrm{w} / \mathrm{w}$ on the powder properties and to compare Cellets with Avicel PH-102 (MCC powder).

\section{Materials and methods}

\subsection{Materials}

Inert spherical particles of MCC (Cellets 90, 100, 200 and 350; manufactured by IPC, Process-Center $\mathrm{GmbH}$ \& Co KG, Germany) ware kindly provided by HARKE Pharma GmbH (Germany). The MCC powder (Avicel PH-102) was supplied as a gift by IMCD UK Ltd. (United Kingdom). Magnesium stearate (MgSt) was gifted by excipient manufacturer Sudeep Pharma Pvt. Ltd. (India).

\subsection{Methods}

\section{Moisture content}

The moisture content of materials was determined via loss on drying (LOD) of approximately $1 \mathrm{~g}$ of pellets samples after equilibration at $105^{\circ} \mathrm{C}$ drying temperature (moisture analyser MB45, Ohaus Corp., Switzerland).

\section{Particle size distribution (PSD)}

The PSD of Avicel PH-102 and MgSt was measured using laser diffraction (LD; ASPIROS dosing, RODOS dispersing at 2 bar, and HELOS / KF LD-detector; Sympatec GmbH, Germany). The R5 lens $(4.5-875 \mu \mathrm{m}$ detection range) was used. The average particle diameter was calculated using largest and smallest 2D-dimension of every Cellet (more than 1000 particles in total) measured using a digital microscope VHX-600 Series (Keyence Corp., Osaka, Japan). The $\mathrm{D}_{10}, \mathrm{D}_{50}$ and $\mathrm{D}_{90}$ were extracted from the cumulative volume 
104

105

106

107

108

fraction which was generated from the raw data. The Span was used as an indicator of particle size distribution and calculated using the following equation:

Span $=\frac{D_{90}-D_{10}}{D_{50}}$

\section{Equation 1}

\section{Determination of particles density}

The density of microparticles was determined using the measurement of particle diameter $(\mathrm{n}=30)$ with a light microscope (and following calculation of volume), the direct measurement of particle sedimentation in water at room temperature and calculation of the particle density in accordance with the modified Stokes' Law equation:

$\rho=\frac{V_{t} \times 18 \eta}{D^{2} \times g}+\rho_{0}$

Equation 2

where: $\rho$ and $\rho_{0}$ - density of spherical particle and water; $\eta$ - viscosity of water; $V_{t}-$ sedimentation velocity; $D$ - diameter of spherical particle; $g$ - gravitational acceleration.

Theoretical calculation of one spherical particle mass and apparent specific surface area $\left(S S A_{A P P}\right)$

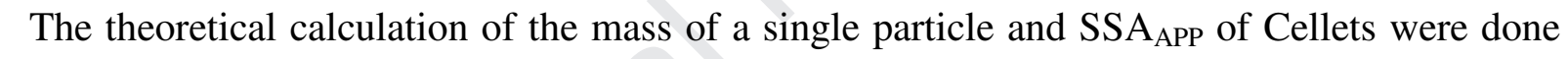
using the assumption that particles have an ideal spherical shape and non-hollow, non-porous structure. For every particle size, the volume (V) and mass (m) was calculated using the following equations:

$V=\frac{4}{3} \pi\left(\frac{D_{50}}{2}\right)^{3}$

Equation 3

$m=\rho V$

Equation 4

using the true density $(\rho)$ of Cellets $350\left(1.44 \mathrm{~g} / \mathrm{cm}^{3}\right.$ with standard deviation $\pm 0.03 \mathrm{~g} / \mathrm{cm}^{3}$; in accordance to Equation 2).

The surface area (A) of a single spherical particle was calculated using:

$A=4 \pi\left(\frac{D_{50}}{2}\right)^{2}$

Equation 5

while $\mathrm{SSA}_{\mathrm{APP}}$ was calculated by taking into the account the number of spherical particles in $1 \mathrm{~g}(\mathrm{~N})$ using:

$S S A_{A P P}=A N$

Equation 6

Specific surface area determination $\left(S S A_{B E T}\right)$ 
$\mathrm{SSA}_{\mathrm{BET}}$ analysis was conducted using an Inverse Gas Chromatography Surface Energy Analyser (iGC-SEA, Surface Measurement Systems Ltd, UK). Approximately 1.8-2 g of each sample was packed into silanised iGC glass columns (internal diameter $4 \mathrm{~mm}$ ). Prior to any measurements, the columns were conditioned using helium carrier gas at $10 \mathrm{scc} / \mathrm{min}$ for $2 \mathrm{~h}$ at $30^{\circ} \mathrm{C}$ and $0 \% \mathrm{RH}$. Methane gas was injected at the start and the end of the experiments

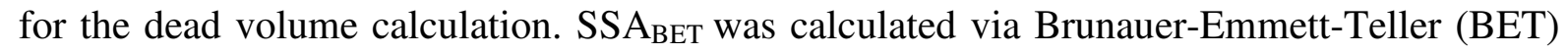
theory, based on the n-octane adsorption isotherm data (Peak Max parameter) [11, 12]. Every sample was analysed in triplicate. A correlation coefficient $\left(R^{2}>0.999\right)$ assured linearity within the BET range.

\section{Mixing with magnesium stearate}

Before mixing, MgSt was passed through a sieve with $0.5 \mathrm{~mm}$ mesh. Then, MgSt was added to Cellets or Avicel PH-102 and mixed in a Turbula mixer (Turbula T2F, Willy A. Bachofen AG, Switzerland) for a 5 min.

\section{Mass flow rate determination with gravitational funnel method}

A stainless-steel frustum cone funnel was fixed in a strictly vertical position (Flowability Tester model BEP2; Copley Scientific Ltd., England). $50 \mathrm{~g}$ samples were weighed and introduced carefully into the dry funnel with $5 \mathrm{~mm}$ diameter orifice. During filling, the bottom opening of the funnel was closed. Once the funnel was opened the time taken for the $50 \mathrm{~g}$ of powder within the funnel to flow out was measured $(\mathrm{n}=6)$. The mass flow rate was calculated as mass per time, expressed in g/s. [13]

\section{Bulk and tapped density testing (pharmacopoeia method)}

To investigate the bulk and tapped density of microparticles alone and with a glidant, the Tapped Density Tester (Copley Scientific JV1000; Copley Scientific Ltd, England) was used. The volume was visually recorded every 3 taps until 33, then at 66, 100, 1000 and 2000 taps. The bulk and tapped density of samples were investigated using a $50 \mathrm{ml}$ graduated volumetric cylinder with $22 \mathrm{~mm}$ internal diameter. All measurements were made in triplicate. Bulk and tapped densities ( $\rho_{\text {bulk }}$ and $\rho_{\text {tapped}}$, respectively) as well as Hausner ratio (HR) were calculated using the following equations:

$\rho_{\text {bulk }}=\frac{\text { Powder mass }}{\text { Initial volume }}$

Equation 7

$\rho_{\text {tapped }}=\frac{\text { Powder mass }}{\text { Volume after } 2000 \text { taps }}$

Equation 8 
$H R=\frac{\rho_{\text {tapped }}}{\rho_{\text {bulk }}}$

\section{Equation 9}

162

163

164

165

166

167

168

169

170

171

172

173

174

175

176

177

178

179

180

181

182

183

184

185

186

187

188

189

190

191

192

\section{The dynamic cohesive index and dynamic angle of repose determination}

The dynamic cohesive index and dynamic angle of repose were measured with a Rotating Drum tester (GranuDrum, GranuTools sprl, Belgium). Approx. 50-60 ml of microparticles were placed into a stainless-steel cylinder (internal diameter $84 \mathrm{~mm}$, length $20 \mathrm{~mm}$ and approx. internal volume $111 \mathrm{ml}$ ) with round glass side walls that have an anti-sticking internal surface. The cylinder was installed horizontally, rotated around its axis at an angular velocity $(2-50 \mathrm{rpm})$ starting off the flowing motion of the microparticle sample being tested. The rotated drum was backlit and for every rotation speed 50 images of the drum separated by $0.5 \mathrm{~s}$ were recorded with a CCD camera. Obtained images were used as raw data and the position of air/powder interface was determined automatically due to the difference in the light intensity. Then, the average air/powder interface position and the fluctuation around this average position as standard deviation $\left(\sigma_{\mathrm{f}}\right)$ were automatically computed using 50 images at every rotation speed. All measurements were made in triplicate. The angle of the average air/powder interface position regarding the horizontal axis was considered as the dynamic angle of repose. The dynamic cohesive index $\left(\sigma_{\mathrm{f}}\right.$, expressed in $\left.\%\right)$ is the deviation from average steady flow and can be used for quantification of cohesion between the microparticles. The fluctuation of air/powder interface and consequently the dynamic cohesive index increases with increasing of particle cohesivity. [14]

Conditioned Bulk Density, Basic Flowability Energy and Specific Energy measurements were performed using the FT4 Powder Rheometer ${ }^{\circledR}$ (Freeman Technology Ltd, England). All samples for dynamic tests were conditioned in the test-vessel using the instrument's "conditioning" methodology: the twisted blade's action gently disturbs the microparticle bed and creates a uniform, lightly packed test sample that can be readily reproduced. After the conditioning cycle, the Conditioned Bulk Density $(\mathrm{g} / \mathrm{ml})$ of the conditioned microparticle bed in the $25 \mathrm{ml}$ cylindric split borosilicate test vessel (with internal diameter $25 \mathrm{~mm}$ ) was recorded. To determine the Basic Flowability Energy (BFE, mJ) the conditioned microparticle bed was consolidated by a bulldozing blade (with external rotational diameter $23.5 \mathrm{~mm}$ ) action that forces the microparticles downwards towards the bottom of the test vessel (where the base of the vessel is limits the powder's movement) at a constant flow rate (anticlockwise blade tip speed of $100 \mathrm{~mm} / \mathrm{s}$ ). The BFE is a key flowability parameter and is calculated as the required energy for the blade to pass through the sample in this downward 
193

194

195

196

197

198

199

200

201

202

203

204

205

206

207

208

209

210

211

212

213

214

215

216

217

218

219

220 movement and provides an indication of the resistance to the blade's movement from the powder.

The Specific Energy (SE, mJ/g) was measured and calculated from the work done in moving the same blade through the microparticle bed from the bottom of the vessel to the top (upward clockwise motion of blade). This generates a gentle lifting and low stress flow of the microparticles, measuring the level of mechanical interlocking, and inter-particle friction. All measurements were made in triplicate.

\section{Aeration test (Aerated Energy measurement)}

The presence or absence of air in a powder can greatly affect its flow properties. The addition of air can occur naturally when powder is moved freely, e.g. when discharging powder from a hopper. When a powder is aerated, the twisted blade encounters less resistance than in the non-aerated state as it passes through the powder bed. The Aerated Energy is measured using the same blade as is used during BFE measurements, and the blade follows the same downwards, anticlockwise motion through the microparticle bed.

\section{Bulk property measurements with powder rheology: Pressure Drop, Permeability,}

\section{Compressibility}

Bulk measurements were performed with FT4 Powder Rheometer (Freeman Technology Ltd, England). A porous vented piston (with external diameter $24 \mathrm{~mm}$ ) was used to apply increasing levels of normal stress on $10 \mathrm{ml}$ of microparticles in a cylindric split borosilicate test vessel (with internal diameter $25 \mathrm{~mm}$ ). The automatically computed volume changes after applied levels of normal stress were used for Compressibility (\%) calculation with the following equation:

Compressibility $\%=\frac{\text { Initial volume-Compressed volume }}{\text { Initial volume }} 100 \%$;

Equation 10

The pressure drop $(\Delta \mathrm{P}$, mbar) across the microparticle bed was recorded whilst the applied normal stress was varied and the air flow through the pellets' bed was maintained at a constant velocity. From $\Delta \mathrm{P}$, using Darcy's Law (Eq. 11), rearranging and dividing by the area the Permeability $\left(\mathrm{k}, \mathrm{cm}^{2}\right)$ can be calculated (Eq. 12):

$Q=\frac{k A}{\mu} \frac{P_{a}-P_{b}}{L}$

Equation 11 
$224 \quad k=\frac{q \mu L}{\Delta P}$

\section{Equation 12}

225 where, q is flux, or air flow rate $(\mathrm{cm} / \mathrm{s})$ and $\mu$ is $1.74 \times 10-7$ (mbar $\cdot \mathrm{s})$ for air at sea level.

226

227

where: $Q$ is Air volume per unit time $\left(\mathrm{cm}^{3} / \mathrm{s}\right) ; A$ is cross-sectional area of powder bed $\left(\mathrm{cm}^{2}\right)$; $(P a-P b)=\Delta P$ is pressure drop across powder bed $(\mathrm{Pa}) ; \mu$ is air viscosity $(\mathrm{Pa} \cdot \mathrm{s}) ; L$ is length of powder bed $(\mathrm{cm})$.

Due to the significant differences in the permeability of MCC samples, it was impossible to obtain comparable permeability measurements at the same conditions. So, Cellets 90 and 200 were tested at $10 \mathrm{kPa}$ normal stress and $2 \mathrm{~mm} / \mathrm{s}$ air velocity while Cellets 100, 200, 350 and Avicel PH-102 were tested at $10 \mathrm{kPa}$ and $20 \mathrm{~mm} / \mathrm{s}$. To provide comparable data at $20 \mathrm{~mm} / \mathrm{s}$ air velocity a conversion calculation was used:

$\Delta P($ Cellets $90 @ 20 \mathrm{~mm} / \mathrm{s})=\frac{\Delta P(\text { Cellets } 90 @ 2 \mathrm{~mm} / \mathrm{s})}{\Delta P(\text { Cellets } 200 @ 2 \mathrm{~mm} / \mathrm{s})} \times \Delta P($ Cellets $200 @ 20 \mathrm{~mm} / \mathrm{s}) ;$ Equation 13

Note that this calculation assumes a linear relationship between Pressure Drop and Air Velocity. All measurements were made in triplicate.

\section{Results}

The particle size of Cellets was measured with an optical digital microscope to avoid speculation arising from traditional light scattering methods on the measured values. The particle size $\left(D_{50}\right)$ values of the Cellets 90, 100, 200 and 350 are 94, 163, 270 and $424 \mu \mathrm{m}$, correspondently (Figure 2, Table 1). Particle size distribution was characterised by narrow span values suggesting a relatively uniform size distribution. Cellets' sphericity increased with increasing particle size (Figure 3).

The increase in Cellets' size was accompanied with a decrease in the calculated SSA $\mathrm{APP}_{\mathrm{A}}$ (Table 1, Figure 4). Thus, an increase in mechanical interlocking with decrease in the size of the Cellets was expected. The calculated $\mathrm{SSA}_{\mathrm{APP}}$ values were much lower than the

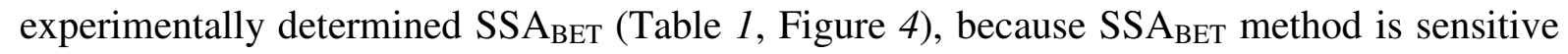
to the particle's surface roughness. SSA $\mathrm{APP}_{\text {and }} \mathrm{SSA}_{\mathrm{BET}}$ of Cellets 90 and Cellets 100 both show a decrease in surface area with increasing particle size. The difference between SSA of Cellets 100 and Cellets 200 was relatively low and can be attributed to the detection limits of the iGC [12]. Despite the relatively similar particle size of Cellets 90 and Avicel PH-102,

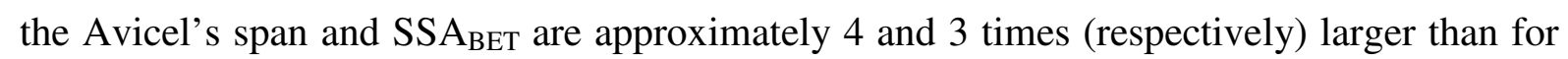
Cellets 90 (Table 1). 
251 The bulk and tapped density (Table 1, Figure 5) of the Cellets are approximately 2 times

252

253

254

255

256

257

258

259

260

261

262

263

264

265

266

267

268

269

270

271

272

273

274

275

276

277

278

279

280

281 higher than for Avicel PH-102. So, Avicel PH-102 powder had higher SSA $\mathrm{BET}_{\text {and }}$ was less efficiently packed than Cellets. The bulk and tapped density of Cellets increased with increasing particle size: Cellets $90<$ Cellets $100<$ Cellets $200<$ Cellets 350. The densification kinetics of the different grades of Cellets was approximately the same (Figure 5) with the fastest densification kinetics observed during first 3-12 taps. From a practical point of view, this suggests that even a few periodical vibrations/oscillations (such as impacts with a rubber hammer) during the coating process could significantly change the density of Cellets in the down-flow bed.

Mixing Cellets with MgSt improved the packing of the particles and decreased the necessary volume for the same number of particles. Thereby increasing the tapped density of Cellets (Figure 5) without significantly impacting the densification kinetics. The tapped density difference between Cellets 90, Cellets 100 and Cellets 200 mixed with MgSt was very low compared to the significant difference without $\mathrm{MgSt}$. The increase in the Cellets' tapped density after adding of $\mathrm{MgSt}$ is likely to be due to a decrease in interparticle friction because of the MgSt's lubrication properties.

The additional densification of Cellets was investigated by applying a normal force. At the same applied force, the compressibility of Cellets decreases as particle size increases (Figure 6, Table 2). Avicel PH-102 also demonstrated a decrease in compressibility upon the addition of MgSt. Interestingly, the compressibility of Cellets with MgSt was very similar irrespective of the particle size. This is most likely due to a decrease in frictional forces arising from the lubrication by MgSt.

The decrease in the pressure drop from Cellets 90 to Cellets 350 (Figure 7 A, Table 2) and corresponding increase in permeability (Figure 7 B, Table 2), alongside the decrease in compressibility indicates that there is a worsening particle packing efficiency (increase in voids between particles) as the particle size increases from Cellets 90 to Cellets 350. Mixing the Cellets with $0.5 \% \mathrm{MgSt}$ didn't change the trends in pressure drop and permeability but decreased the compressibility, indicating that there was a tighter, more efficient particle packing (Figure $7 \mathrm{~A}$ and B, Table 2).

The gravitational funnel method suggests an absence of correlation between the Cellets flow properties and their size (Figure $8 \mathrm{~A}$ ) or $\mathrm{SSA}_{\mathrm{APP}}$ (Figure $8 \mathrm{~B}$ ). Notably, in contrast to the 
similarly sized Cellets 90, the Avicel PH-102 did not flow through the funnel with $5 \mathrm{~mm}$ diameter-opening.

The dynamic angle of repose measured with a rotating drum tester gives information regarding the dynamic ability of powders to flow. This could be useful for predicting, to some extent the powders ability to be conveyed or gravitationally discharged. The measurement of dynamic angle of response showed differences between the relatively poor flowing Avicel PH-102 and better flowing Cellets. However, the difference between different grades of Cellets wasn't clear (Figure 9 A).

SE measurements, using the FT4 powder rheometer, gives information regarding the level of interlocking and friction between powder particles, which will also inform on how a powder may flow under gravity, i.e. its resistance to flow in an unconstrained environment. SE values of Cellets without MgSt increased with respect to decreasing particle size (Figure $9 \mathrm{~B}$, Table 3). The increasing of Cellets sphericity (Figure 3) and decreasing of SSA (Figure 4) as well as decreasing of Cellets' number (Cellets/g) with increasing particle size are suggestive of a decrease in the level of mechanical locking and surface of interparticle friction. So, it can explain the SE decreasing with particle size increase. The addition of MgSt decreased the SE for all Cellets' sizes and decreased the difference between them. This information is in line with the previously shown densification and compressibility measurements, where it was concluded that the addition of MgSt resulted in an increase in the packing efficiency. The greatest SE value was observed for Avicel PH-102, indicating high interparticle friction due to high surface area and roughness, irregular shape and mechanical interlocking, which also decreased with the addition of MgSt (Figure 9 B, Table 3).

The BFE is a measure of the resistance required by a twisted blade to displace a powder during non-gravitational, forced flow, i.e. its resistance to flow in a constrained environment, for example, feeding via flowing in a conveyer [15] or mixing [16]. Cellets without $\mathrm{MgSt}$ demonstrated an almost linear increase in BFE with decreasing size (Figure $10 \mathrm{~A}$ ). While Cellets mixed with MgSt showed a decrease in BFE for every Cellets' size, the addition of MgSt almost neutralised the BFE difference between the various particle sizes. As with the Cellets, the BFE of Avicel PH-102 with MgSt was lower than for Avicel PH-102 without MgSt. The BFE of Avicel PH-102 with and without MgSt was lower than the lowest value of all tested Cellets with and without MgSt (Figure $10 \mathrm{~A}$ ), that is apparently this is due to the relatively low density of Avicel PH-102. 
314 The Aeration test measures changes in the flow properties due to the introduction of air into

315 the sample. With increasing levels of air velocity (Figure $10 \mathrm{~B}$ ) there is a decrease in the aerated energy for all the samples, indicating that the addition of air has some influence during processing on all the samples, with and without MgSt. Cellets 90 and Avicel PH-102 both with and without $\mathrm{MgSt}$ had similar responses to being aerated and were completely fluidised at $8 \mathrm{~mm} / \mathrm{s}$ air velocity. Cellets 100 with and without $\mathrm{MgSt}$ fluidised at $16 \mathrm{~mm} / \mathrm{s}$ air velocity. Cellets 200 with MgSt were completely fluidised at $40 \mathrm{~mm} / \mathrm{s}$ while Cellets 200 without MgSt weren't. The addition of MgSt on the Aerated Energy has had a similar impact on both the Cellets 200 and 350, where the Aerated Energy at $40 \mathrm{~mm} / \mathrm{s}$ air velocity has decreased by 26.5 and $23.5 \mathrm{~mJ}$ respectively (Figure $10 \mathrm{C}$ ). The Aeration test can determine the powder's Flow Energy with increasing air velocity, and is a measure of the powder's cohesivity, as well as the minimum fluidisation velocity. The Aeration test clearly showed that the minimum fluidisation velocity of Cellets' sizes increased with increasing particle size, and the cohesivity of Cellets and Avicel PH-102 decreased with the addition of MgSt.

For the characterisation of cohesivity in another way, Cellets' dynamic cohesive index was determined with the rotating drum tester. The measurement of dynamic cohesive index showed a decrease in cohesivity in the following sequence: Avicel PH-102 > Cellets $90>$ Cellets $100>$ Cellets $200 \approx$ Cellets 350 (Figure 11). Comparing to the Aeration test (Figure $10 \mathrm{~B})$, the rotating drum tester has a limited extent to which it can determine the cohesivity differences between Cellets 200 and Cellets 350 .

\section{Discussion}

Usually, cores with particle size larger than $350 \mu \mathrm{m}$ (e.g. Cellets 350 with $\mathrm{D}_{50} 424 \mu \mathrm{m}$ ) don't display any process issues in the Wurster fluid-bed coater. So, considering their properties as appropriate or non-problematic the effect of Cellets' size and addition of MgSt on the powder rheology and possible effect on the coating process can be assessed. Avicel PH-102 has been added to the experimental plan as it widely used in industry and has been used in several investigations and can be used by readers to make connections with other studies. From a range of powder flow measurement techniques, some methods are better explaining Cellets' behaviour in a specific zone of fluid-bed coater's processing chamber while some of them are applicable for several zones. 
344 Down-flow bed zone. The difference in powder behaviour in the down-flow bed zone could be explained using the basic flowability energy (BFE), dynamic cohesive index, densification kinetics, compressibility, pressure drop and permeability measurements.

The BFE measurement can be applicable for the understanding of powder flow in a constricted volume. In this situation, it can be used as indicator of the level of particle mobility and powder rearrangement in the down flow bed zone. An almost linear dependence of the BFE decreasing was shown for Cellets' size $\left(D_{50}\right)$ increasing from 94 to $424 \mu \mathrm{m}$. The BFE is an indicator of sum of different forces, the determination of dynamic cohesive index is elucidating and in some extend representing mostly cohesivity. The trend of cohesive index decreasing with Cellets' size increasing was the same as for BFE but was not able to differentiate between Cellets 200 and 350 .

The results of densification kinetics illustrate the comparison of the interparticle friction and are interesting especially since vibration devices or periodic tapping are used as powder flow facilitation approach during Wurster fluid-bed processing. Compressibility testing, to some extent, could be considered as a continuation of the densification kinetics test with additional vertical axial normal stress. The results of densification kinetics and compressibility measurement were in the agreement with BFE and dynamic cohesive index with an almost linear dependence of a decreasing compressibility with increasing Cellets' size $\left(\mathrm{D}_{50}\right)$ from 94 to $424 \mu \mathrm{m}$.

In the addition to the other methods to characterise powder behaviour in the down-flow bed zone, the pressure drop across the microparticle bed and permeability provide the information regarding the resistance experienced by the Cellets' bed to passing through air and can explain the difference between drying processes in the down-flow bed for Cellets with different particle sizes. The pressure drop decreases, and permeability correspondingly increases as the Cellets' size increases from 94 to $425 \mu \mathrm{m}$. This is suggestive of the drying ability of Cellets in the down-flow bed is improves as Cellets' size increases.

The horizontal transport zone. To understand powder behaviour as it moves through the horizontal transport zone, the mass flow rate through the gravitational funnel, dynamic angle of repose, specific energy and dynamic cohesive index could be used as they are likely to correlate with powder flow under gravity. In addition, the aeration test which is measures the effect of air flow on the resistance of the FT4 blade's motion though the powder particles and could relate to facilitation of particles rearranging. 
Gravitational flow through a funnel did not give any clear correlations between the Cellets particle size and the mass flow rate. While, the SE (which is usually used for characterisation of powder flow in an unconfined process) decreased as the Cellets' size increased however there was a minimal difference between Cellets 200 and 350 (as for dynamic cohesive index mentioned above).

The aeration test provides information on the changing interparticle interactions with increasing air velocity and the minimum fluidisation velocity for different powder samples. The data from the Aeration test could be used to compare and possibly predict the efficiency of powder passing from the horizontal transport zone to the coating zone. The aerated energy (the energy required for the twisted blade to pass through the aerated Cellets') as expected decrease as the air velocity increased for all of the powders tested, and at $40 \mathrm{~mm} / \mathrm{s}$ air velocity, the aerated energy increased as the Cellets' size increased. In other words, as the particle size decreases, or the air velocity increases, the Cellets' ability to be conveyed to coating zone is likely to improve.

The coating zone. Within the coating zone, it is essential that particles are kept separated from each other, to avoid coating-induced agglomeration. The dynamic cohesive index and minimum fluidisation velocity could be correlated to a powder particle's ability to become separated from each other in the coating zone. Measurements of the dynamic cohesive index and minimum fluidisation velocity both assess the particle's cohesivity and in both cases, the particle mass and corresponding gravitational force influence the result. For the dynamic cohesive index measurement, the gravitational force is facilitating the flow and in the case of minimum fluidisation velocity determination, all else being equal, heavier particles are likely to require a higher air velocity.

Initially, both the cohesive index and minimum fluidisation velocity could be directly applied to the powder's behaviour explanation in the coating zone. However, considering the specific details of each measurement, the aeration test which passes air through the powder bed, is most suitable once the effect of gravity on the particles has been counteracted and building a correlation between the atomisation air shear force in the coating zone and determined cohesivity.

Effect of MgSt. The addition of MgSt to Cellets of any particle size and Avicel PH-102 had a lubricating effect, as would be expected from a glidant. The addition of $\mathrm{MgSt}$ at the $0.5 \%$ w/w level increased the tapped density (i.e. increased packing efficiency), decreased BFE, 
$408 \mathrm{SE}$, and aerated energy/ minimum fluidisation velocity. The effect of $\mathrm{MgSt}$ on the 409 gravitational flow through a funnel did not give clear answer, but the applicability of this 410 method to characterise of Wurster fluid-bed process is questionable too (at least with $5 \mathrm{~mm}$ 411 orifice diameter). Generally, almost all flow characterisation methods suggested powder flow 412 improvement in the down-flow bed and horizontal transport zone after adding MgSt. A 413 decrease in the pressure drop and increase permeability after adding MgSt was observed, 414 suggesting that the drying process of lubricated Cellets in the down-flow bed is likely to be 415 slower compared to unlubricated Cellets. This could be correlated to a decrease in the 416 interparticle friction, and generally higher density layer with lower interparticle space in the 417 lubricated Cellets' bed. 


\section{Conclusions}

420

421

422

423

424

425

426

427

428

429

430

431

432

433

434

435

436

437

438

439

440

441

442

443

444

445

446

447

448

449

450

451

452

453

454

455

456

457

The level of Cellets' flowability decreasing in the down-flow bed and horizontal transport zones of Wurster fluid-bed coater with decreasing of Cellets size $\left(\mathrm{D}_{50}\right)$ from 424 to $94 \mu \mathrm{m}$ was established with different powder rheology methods. In the horizontal transport zone, along with decreasing of Cellets' size, the decreasing of Cellets flowability can be to some extent compensated with decrease in the minimum fluidisation velocity and consequent increase in Cellets conveying to the coating zone. While the decrease in Cellets' size decreases the powder flowability in the down-flow and predetermining the powder supply to the horizontal powder zone.

Specific powder rheology methods were proposed for characterisation of Cellets' behaviour in down-flow bed, the horizontal transport and coating zone. The effect of a decrease in the Cellets' particle size is likely to worsen Cellets' flowability and consequently processability in a Wurster fluid-bed coater. Whereas the addition of MgSt (only added at $0.5 \% \mathrm{w} / \mathrm{w}$ ) is likely to improve the powder flow in a Wurster fluid-bed coater.

\section{Literature:}

1. Sidwell, R., et al., Characterization of Inert Cores for Multiparticulate Dosage Forms, in Multiparticulate Drug Delivery. 2017, Springer. p. 5-35.

2. Al-Hashimi, N., et al., Oral Modified Release Multiple-Unit Particulate Systems: Compressed Pellets, Microparticles and Nanoparticles. Pharmaceutics, 2018. 10(4): p. 176 DOI: 10.3390/pharmaceutics10040176.

3. Majekodunmi, S.O., A review on centrifugation in the pharmaceutical industry. Am. J. Biomed. Eng, 2015. 5(2): p. 67-78 DOI: 10.5923/j.ajbe.20150502.03.

4. $\quad \mathrm{Yu}$, L.X., et al., Understanding pharmaceutical quality by design. AAPS J, 2014. 16(4): p. 77183 DOI: 10.1208/s12248-014-9598-3.

5. Lopez, F.L., et al., Effect of formulation variables on oral grittiness and preferences of multiparticulate formulations in adult volunteers. Eur J Pharm Sci, 2016. 92: p. 156-62 DOI: 10.1016/j.ejps.2016.07.006.

6. Christensen, F.N. and P. Bertelsen, Qualitative Description of the Wurster-Based Fluid-Bed Coating Process. Drug Development and Industrial Pharmacy, 1997. 23(5): p. 451-463 DOI: 10.3109/03639049709148494.

7. Börner, M., M. Peglow, and E. Tsotsas, Derivation of parameters for a two compartment population balance model of Wurster fluidised bed granulation. Powder technology, 2013. 238: p. 122-131 DOI: 10.1016/j.powtec.2012.04.014.

8. Hampel, N., et al., Continuous pellet coating in a Wurster fluidized bed process. Chemical Engineering Science, 2013. 86: p. 87-98 DOI: 10.1016/j.ces.2012.05.034.

9. Jiang, Z., et al., Modeling of Particle Behavior in a Wurster Fluidized Bed: Coupling CFD-DEM with Monte Carlo, in Proceedings of 21st International Drying Symposium. 2018 DOI: 10.4995/ids2018.2018.7239. 
10. Mohylyuk, V., et al., Wurster Fluidised bed coating of microparticles: Towards scalable production of oral sustained-release liquid medicines for patients with swallowing difficulties. AAPS PharmSciTech, 2019. XX(XX): p. XX DOI: XXX.

11. Ramachandran, V., et al., Formulation pre-screening of inhalation powders using computational atom-atom systematic search method. Mol Pharm, 2015. 12(1): p. 18-33 DOI: 10.1021/mp500335w.

12. Sharif, S., et al., A simplified approach to determine effective surface area and porosity of low bulk density active pharmaceutical ingredients in early development. Advanced Powder Technology, 2015. 26(2): p. 337-348 DOI: 10.1016/j.apt.2014.11.002.

13. Schussele, A. and A. Bauer-Brandl, Note on the measurement of flowability according to the European Pharmacopoeia. Int J Pharm, 2003. 257(1-2): p. 301-4 DOI: 10.1016/S03785173(03)00142-X.

14. Lumay, G., et al., Measuring the flowing properties of powders and grains. Powder Technology, 2012. 224: p. 19-27 DOI: 10.1016/j.powtec.2012.02.015.

15. Cocco, R., et al., Small-Scale Particle Interactions Are Having Significant Effects on Global Fluidized Bed Behavior. KONA Powder and Particle Journal, 2017. 34: p. 155-167 DOI: 10.14356/kona.2017021.

16. Davis, M.T., C.B. Potter, and G.M. Walker, Downstream processing of a ternary amorphous solid dispersion: The impacts of spray drying and hot melt extrusion on powder flow, compression and dissolution. Int J Pharm, 2018. 544(1): p. 242-253 DOI: 10.1016/j.ijpharm.2018.04.038.

17. Rowe, R.C., P. Sheskey, and M. Quinn, Handbook of pharmaceutical excipients. 6th edn. ed. 2009: Libros Digitales-Pharmaceutical Press. 


\section{Processability $=$ function of bulk powder behaviour $=$ function of Cellets' size}
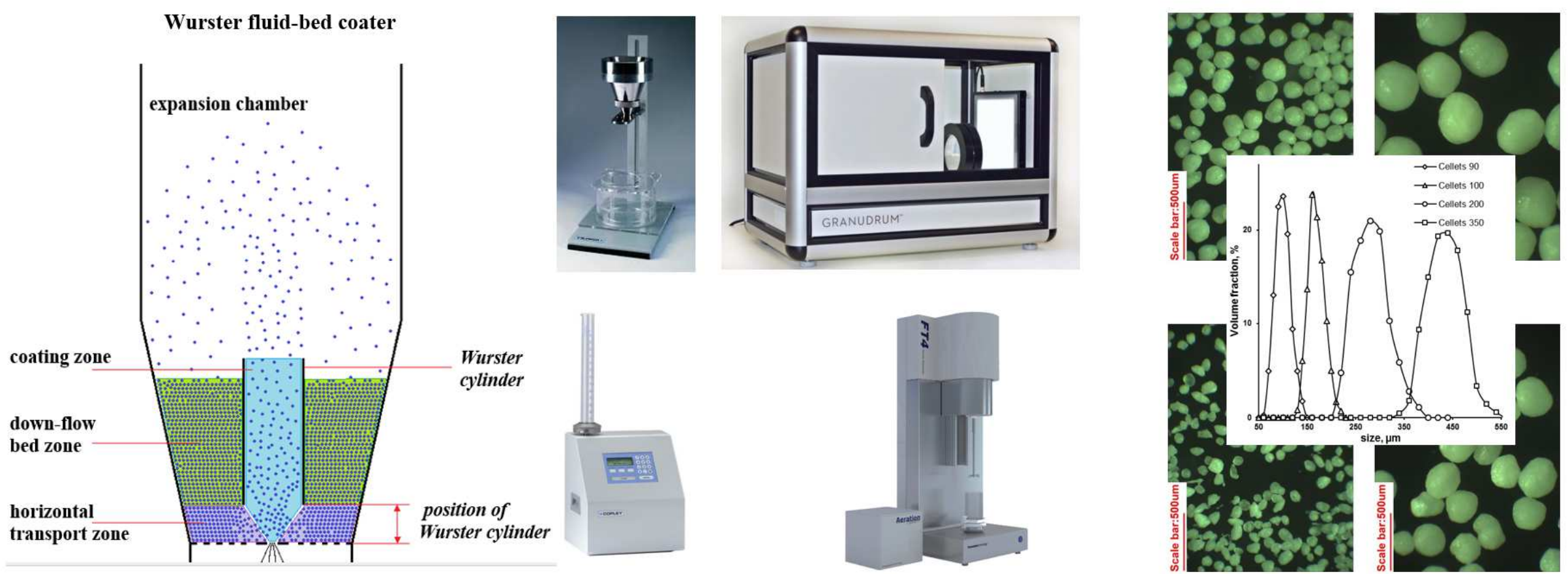
487

488

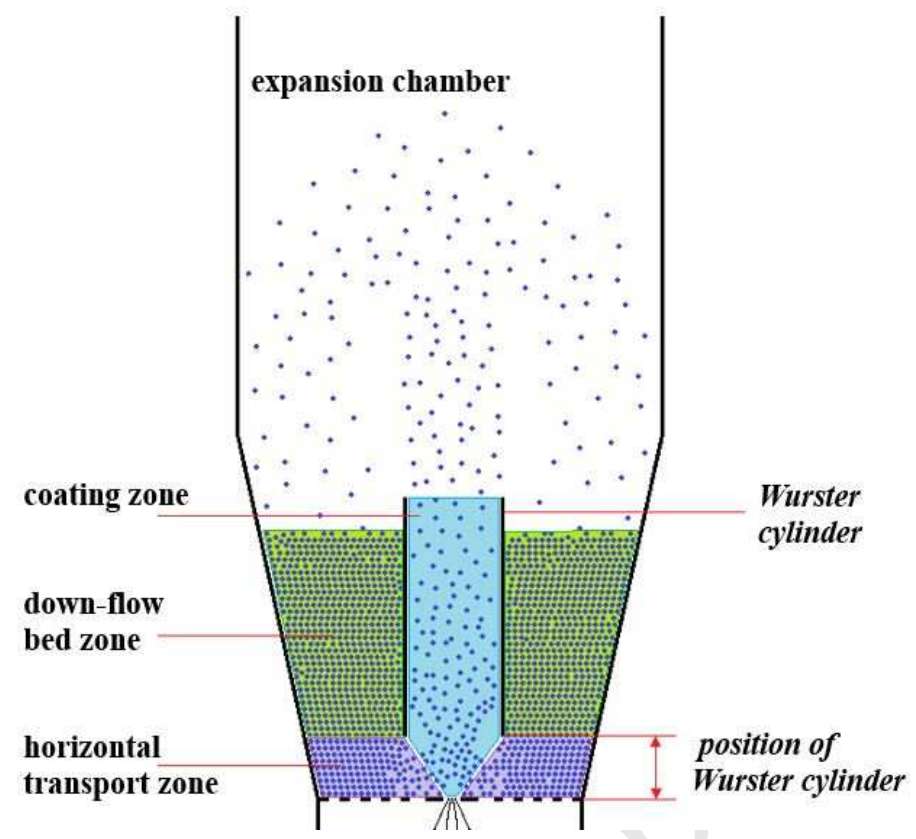

489 Figure 1. Schematic presentation of the working chamber of the Wurster fluid-bed coater. 
491

492

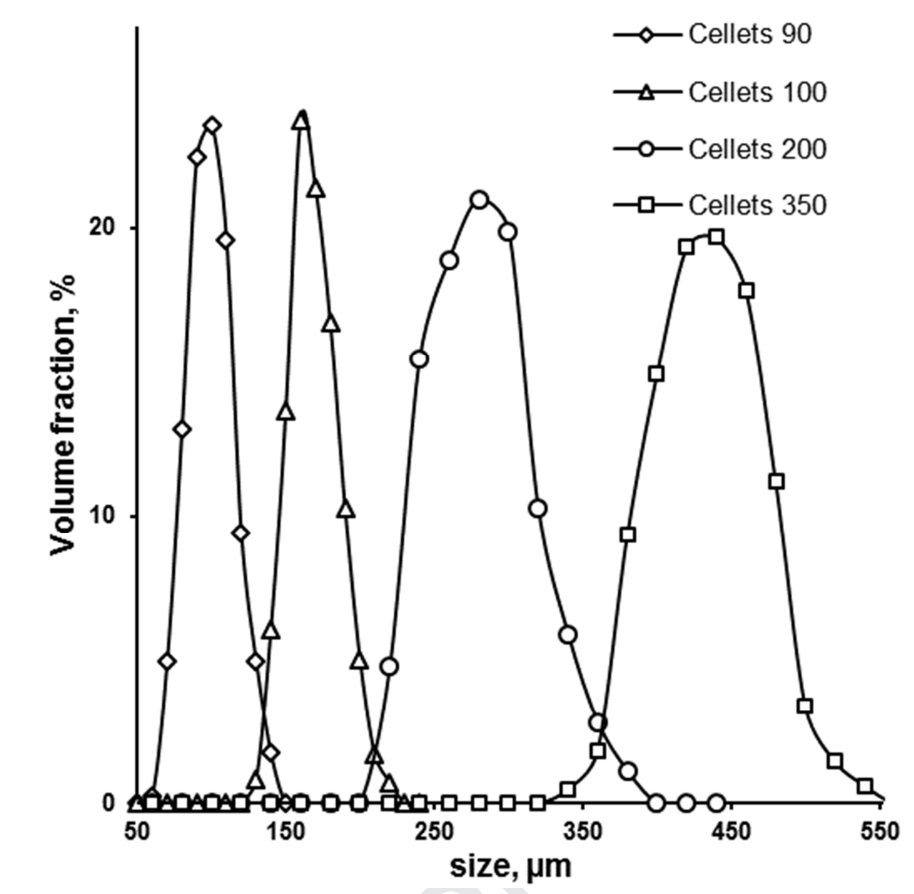

493

494 Figure 2. Particle size distribution of the Cellets used in this study.

495 
497 Table 1. Particle size distribution (PSD), moisture content (LOD method), bulk and tapped density, Hausner ratio and mass flow rate.

\begin{tabular}{|c|c|c|c|c|c|c|c|c|c|c|c|c|c|c|c|c|c|}
\hline & \multirow{3}{*}{ Excipients } & \multicolumn{2}{|c|}{ PSD $^{1}$} & \multicolumn{3}{|c|}{$\mathrm{SSA}, \mathrm{cm}^{2} / \mathrm{g}$} & \multicolumn{2}{|c|}{ LOD, \% (w/w) } & \multicolumn{5}{|c|}{ Density $^{3}, \mathrm{~g} / \mathrm{ml}$} & \multicolumn{2}{|c|}{ Hausner ratio } & \multicolumn{2}{|c|}{ Mass flow rate ${ }^{5}, \mathrm{~g} / \mathrm{s}$} \\
\hline & & \multirow[t]{2}{*}{$\mathrm{D}_{50}, \mu \mathrm{m}$} & \multirow[t]{2}{*}{ Span } & \multirow[t]{2}{*}{$\mathbf{S S A}_{\text {APP }}$} & \multicolumn{2}{|c|}{ SSA $_{\text {BET }}$} & \multirow[b]{2}{*}{ Av } & \multirow[b]{2}{*}{ SD } & \multicolumn{2}{|c|}{ bulk } & \multicolumn{2}{|c|}{$\begin{array}{c}\text { tapped } \\
(2000 \text { taps })\end{array}$} & \multirow[t]{2}{*}{ True $^{4}$} & \multirow{2}{*}{$\begin{array}{c}\text { with- } \\
\text { out } \\
\text { MgSt }\end{array}$} & \multirow{2}{*}{$\begin{array}{c}\text { with } \\
0.5 \% \\
\text { MgSt }\end{array}$} & \multirow[b]{2}{*}{ Av } & \multirow[b]{2}{*}{ SD } \\
\hline & & & & & Av & SD & & & Av & SD & Av & SD & & & & & \\
\hline 1 & $\mathrm{MgSt}$ & $10.5^{2}$ & 2.93 & - & - & - & 3.4 & 0.2 & 0.275 & 0.007 & 0.387 & 0.005 & 1.092 & 1.41 & - & no flow & - \\
\hline 2 & Avicel PH-102 & $115^{2}$ & 1.85 & - & 11033 & 72 & 5.2 & 0.2 & 0.351 & 0.002 & 0.412 & 0.001 & \multirow{4}{*}{$\begin{array}{r}1.460 \\
-1.668\end{array}$} & 1.21 & 1.08 & no flow & - \\
\hline 3 & Cellets 90 & 94 & 0.44 & 433 & 3274 & 23 & 4.9 & 0.1 & 0.750 & 0.004 & 0.796 & 0.003 & & 1.09 & 1.07 & 1.76 & 0.09 \\
\hline 5 & Cellets 200 & 270 & 0.34 & 152 & 1133 & 2 & 4.1 & 0.1 & 0.809 & 0.003 & 0.875 & 0.003 & & 1.11 & 1.06 & 1.89 & 0.01 \\
\hline 6 & Cellets 350 & 424 & 0.22 & 97 & - & - & 4.0 & 0.2 & 0.833 & 0.005 & 0.906 & 0.004 & & 1.10 & 1.09 & 1.83 & 0.01 \\
\hline
\end{tabular}

$498{ }^{1}$ - digital microscopy; ${ }^{2}$ - measured by laser diffraction method; ${ }^{3}$ - tapped density test; ${ }^{4}-$ True density [17]; ${ }^{5}-$ gravitational funnel method; 
500

A) Magnesium stearate

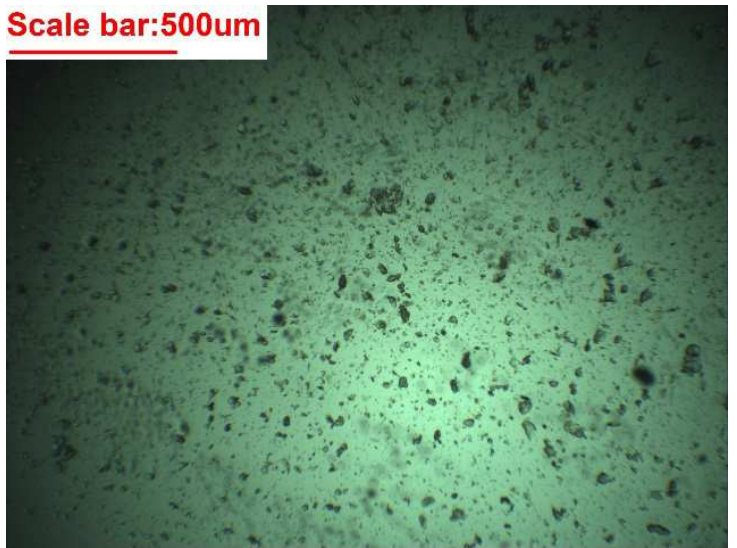

C) Cellets 90

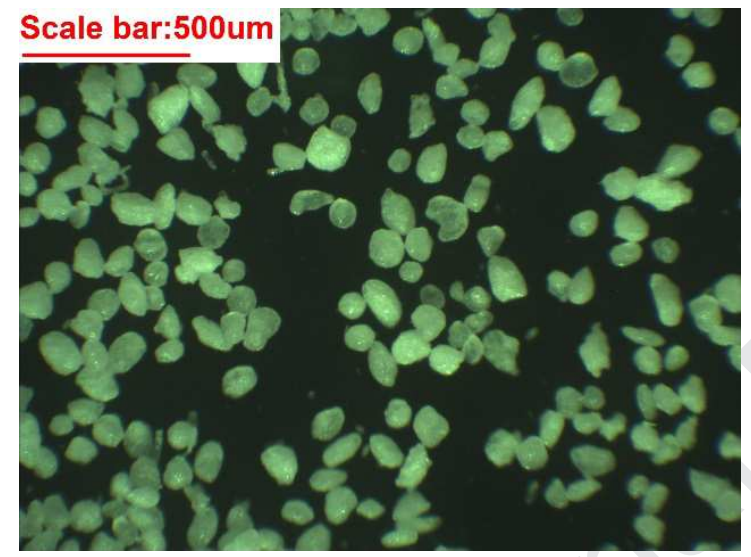

E) Cellets 200

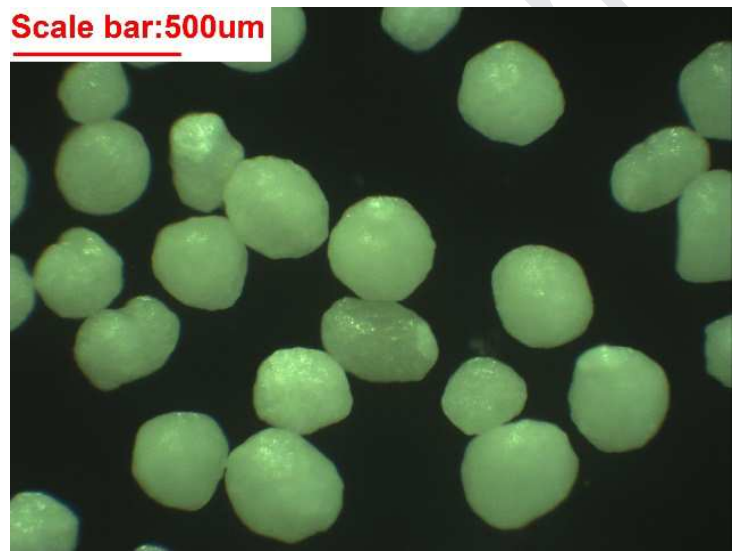

B) Avicel PH-102

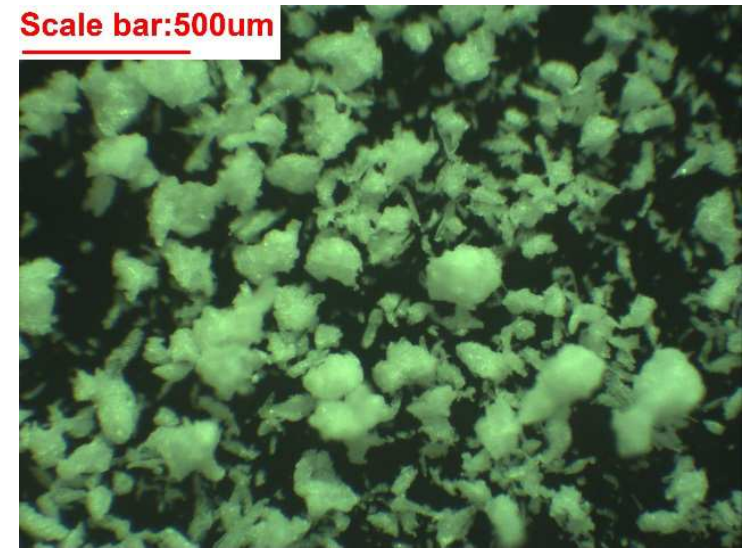

D) Cellets 100

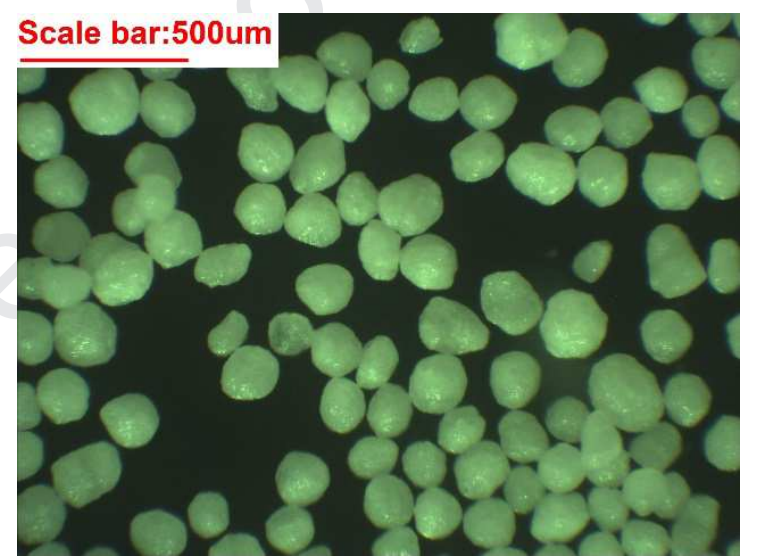

F) Cellets 350

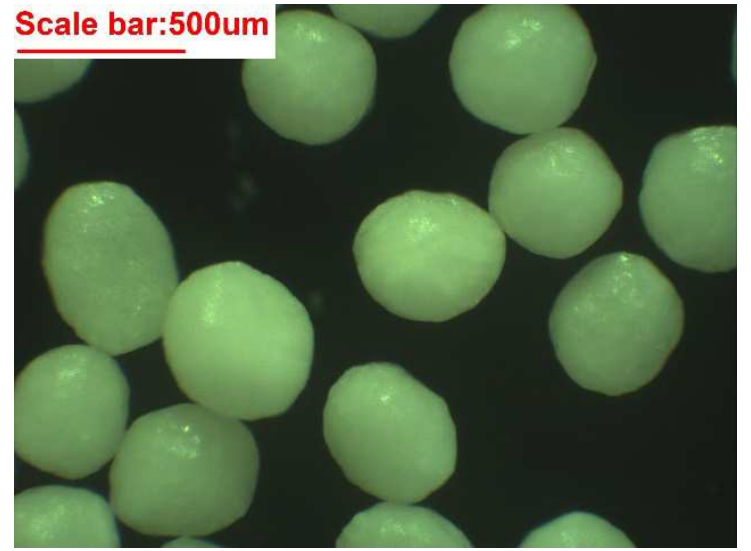

501 Figure 3. Optical microscopy images of excipients: A) magnesium stearate (in liquid 502 paraffin); B) Avicel PH-102; C-F) Cellets 90, 100, 200 and 350, respectively. 
504

$$
\text { - - - single microparticle mass SSA (BET) - SSA (APP) }
$$

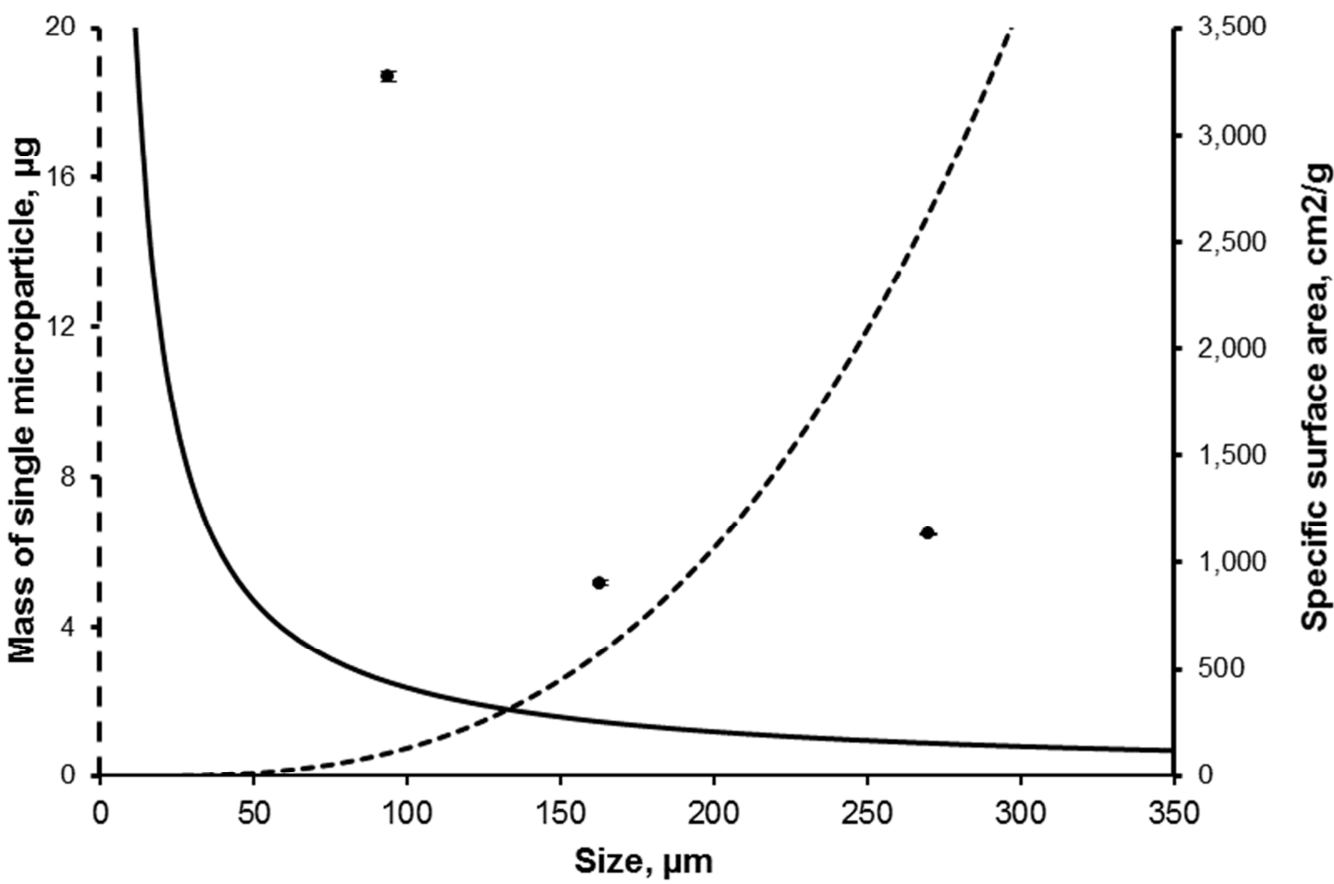

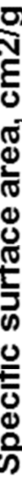

505

506 Figure 4 . The effect of particle size $\left(D_{50}\right)$ on the calculated single microparticle mass and

507 apparent specific surface area $\left(\mathrm{SSA}_{\mathrm{APP}}\right)$ in comparison to the experimentally measured

$508 \quad \mathrm{SSA}_{\mathrm{BET}}$ 


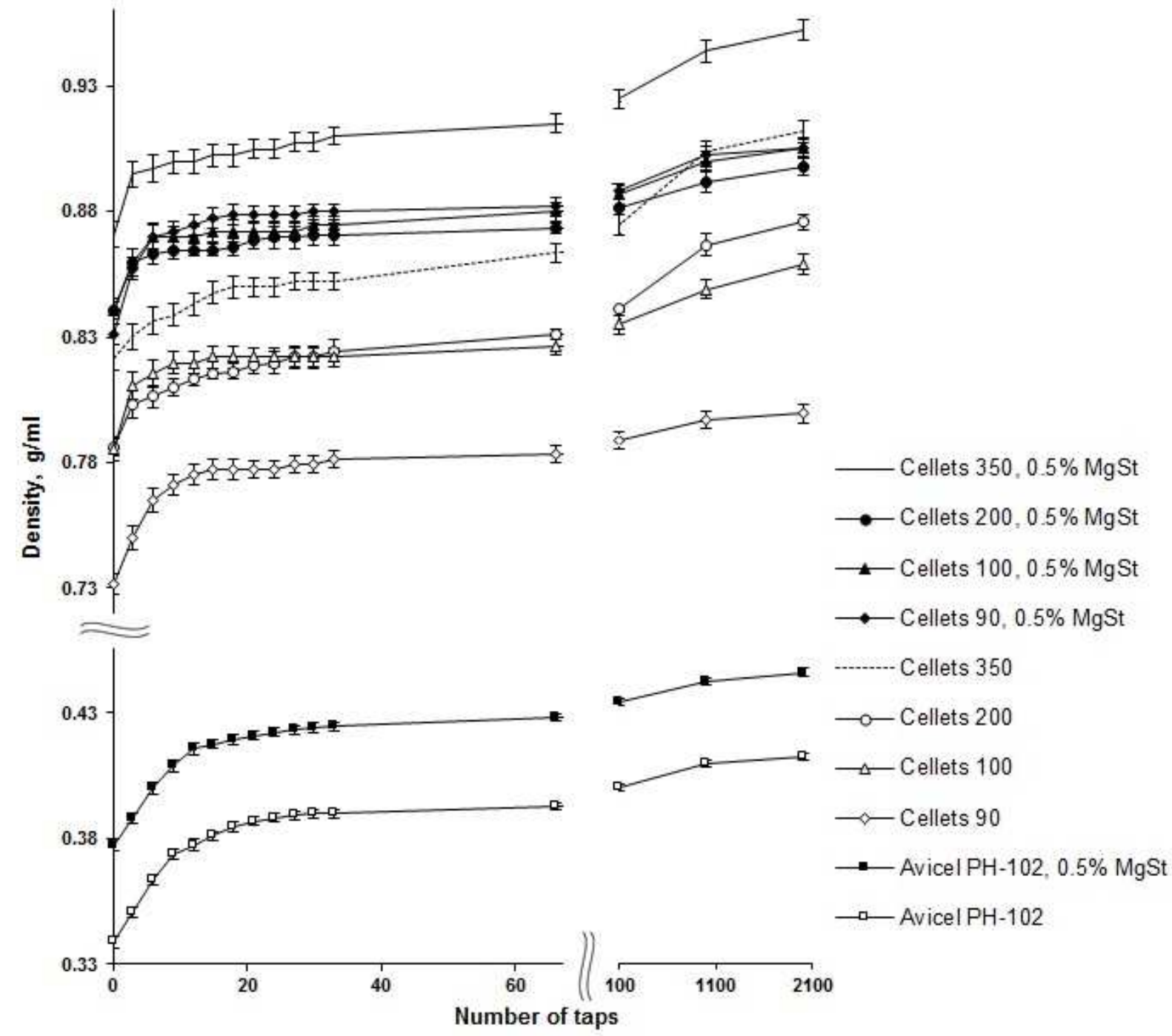

511

512 Figure 5. Densification kinetics of Cellets and Avicel PH-102 without and with $0.5 \% \mathrm{MgSt}$. 


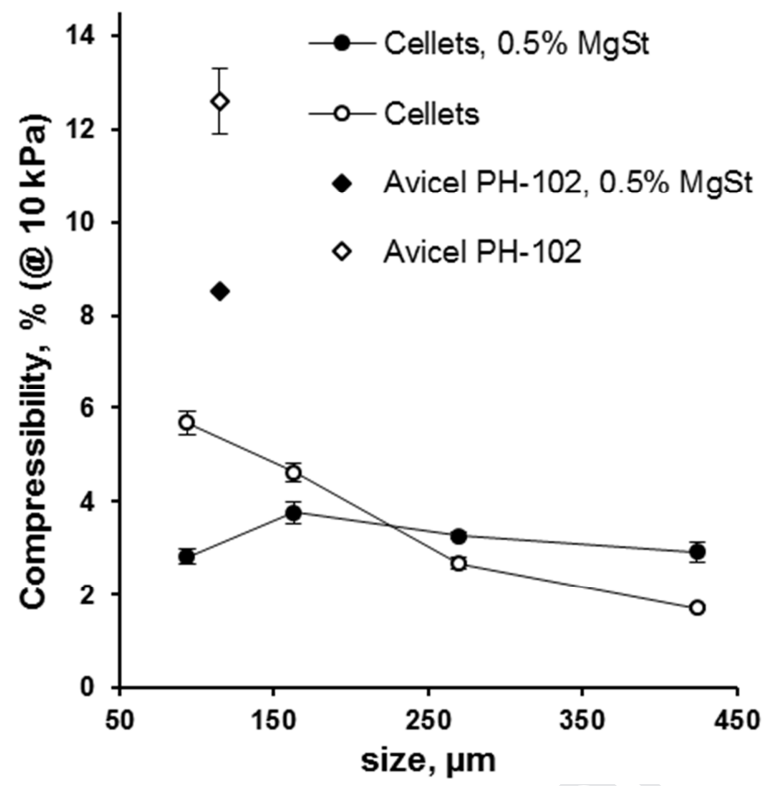

516 Figure 6. Compressibility at $10 \mathrm{kPa}$ normal stress on Cellets with varying particle size and 517 Avicel PH-102, with and without $0.5 \%$ MgSt.

Table 2. Bulk properties of Cellets measured with FT4 powder rheometer at $10 \mathrm{kPa}$ normal stress

\begin{tabular}{|l|c|c|c|c|c|c|}
\hline \multirow{2}{*}{} & \multicolumn{2}{|c|}{ Pressure Drop } & \multicolumn{2}{c|}{ Permeability } & \multicolumn{2}{c|}{ Compressibility } \\
\cline { 2 - 7 } & Av & SD & Av & SD & Av & SD \\
\cline { 2 - 7 } & mbar & mbar & $\mathbf{x 1 0}^{-9} \mathbf{c m}^{\mathbf{2}}$ & $\mathbf{x 1 0}^{-9} \mathbf{c m}^{\mathbf{2}}$ & \% & \% \\
\hline Cellets 350 & 0.6 & 0.0 & 1014.0 & 16.2 & 1.7 & 0.0 \\
\hline Cellets 200 & 1.2 & 0.0 & 467.0 & 25.3 & 2.7 & 0.1 \\
\hline Cellets 100 & 3.6 & 0.1 & 178.0 & 3.0 & 4.6 & 0.2 \\
\hline Cellets 90* & 8.2 & 0.1 & 69.9 & 8.1 & 5.7 & 0.3 \\
\hline Avicel PH102 & 4.7 & 0.1 & 123.0 & 3.3 & 12.6 & 0.7 \\
\hline Cellets 350, MgSt (0.5\%) & 0.7 & 0.0 & 960.0 & 9.8 & 2.9 & 0.2 \\
\hline Cellets 200, MgSt (0.5\%) & 1.8 & 0.0 & 365.0 & 8.4 & 3.3 & 0.1 \\
\hline Cellets 100, MgSt (0.5\%) & 6.1 & 0.0 & 106.0 & 1.1 & 3.8 & 0.2 \\
\hline Cellets 90, MgSt (0.5\%) & 21.3 & 0.1 & 27.2 & 0.1 & 2.8 & 0.2 \\
\hline Avicel PH102, MgSt (0.5\%) & 6.0 & 0.7 & 100.0 & 0.7 & 8.5 & 0.0 \\
\hline
\end{tabular}


A

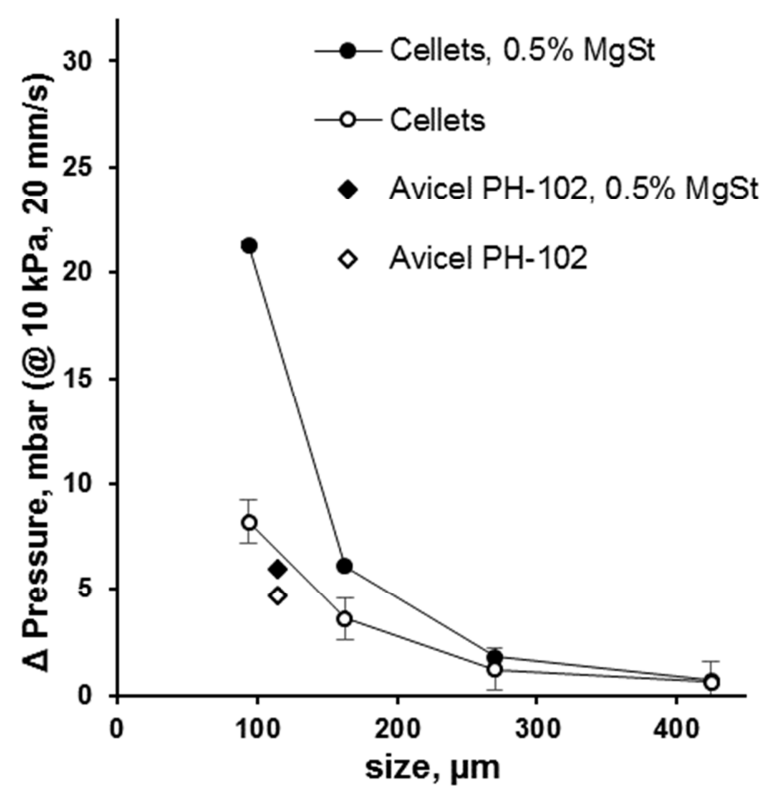

B

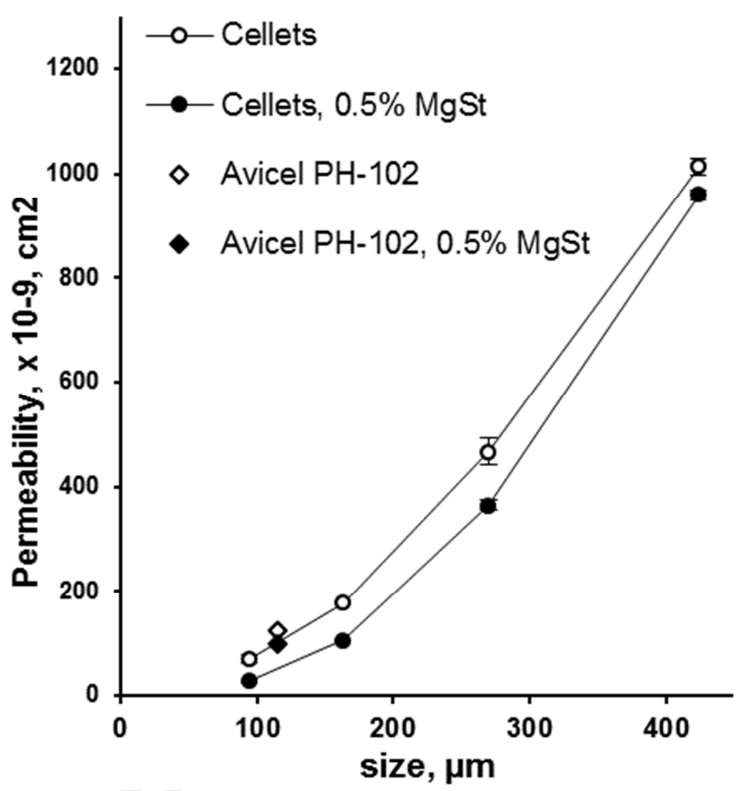

526 Figure 7. Pressure drop $(\Delta \mathrm{P})$ across the microparticle bed (A) and Permeability (B) with 527 increasing Cellets particle size and Avicel PH-102 without and with $0.5 \% \mathrm{MgSt}$. 
A

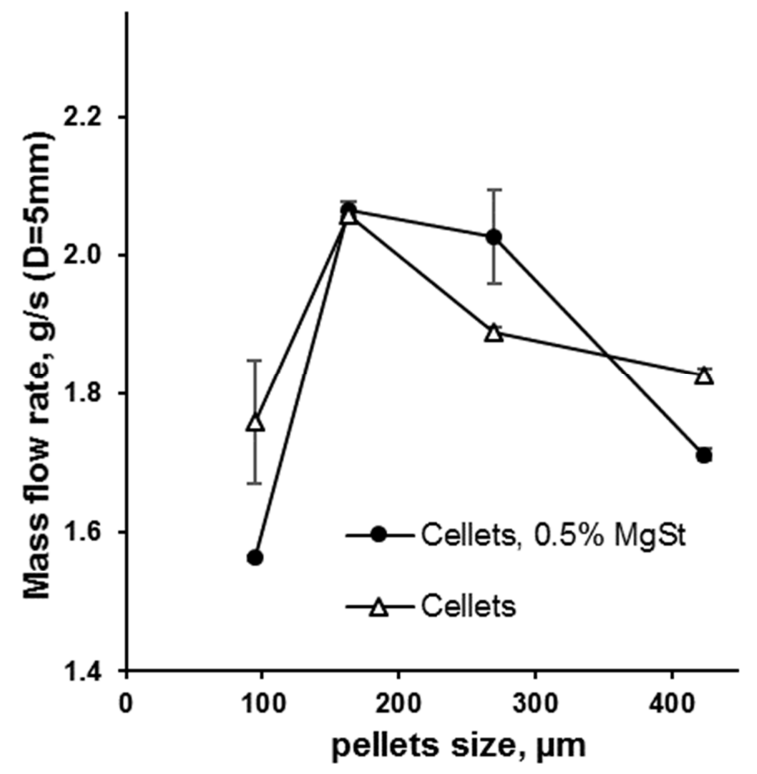

B

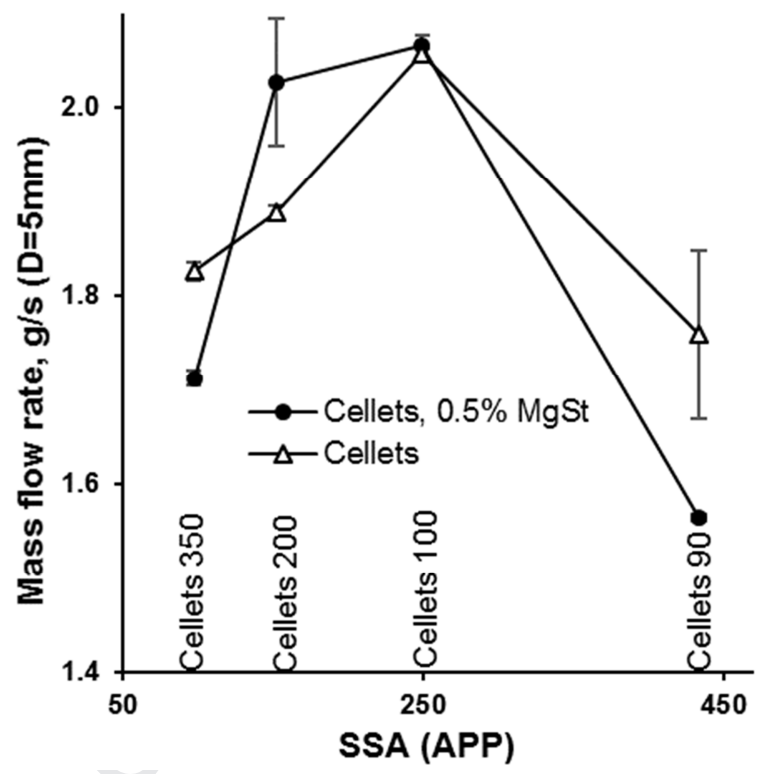

530 Figure 8. Effect of Cellets' size (A) and apparent specific surface area (SSA APP) of Cellets 531 (B) on the mass flow rate without and with $0.5 \% \mathrm{MgSt}$. 
A

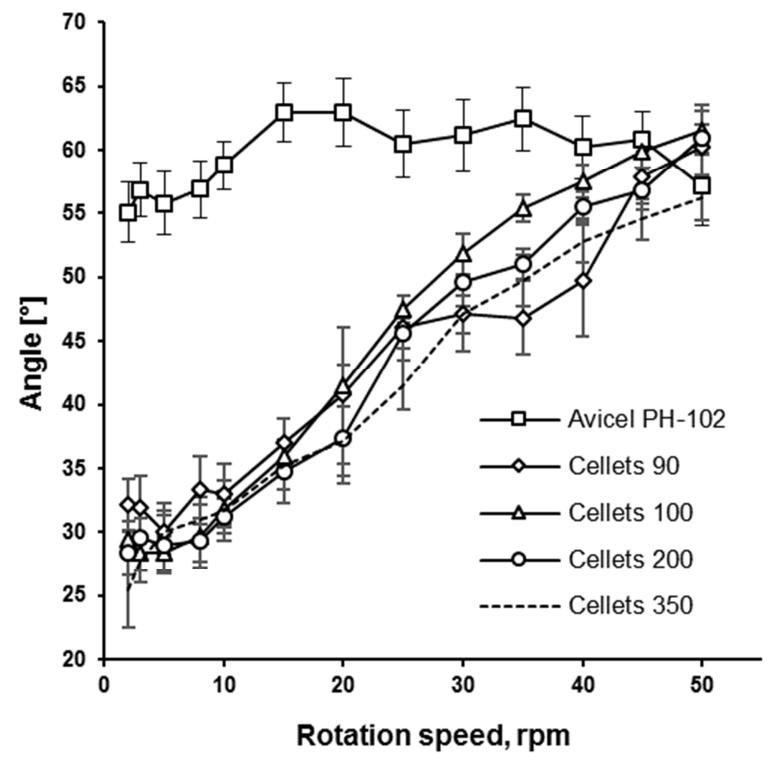

B

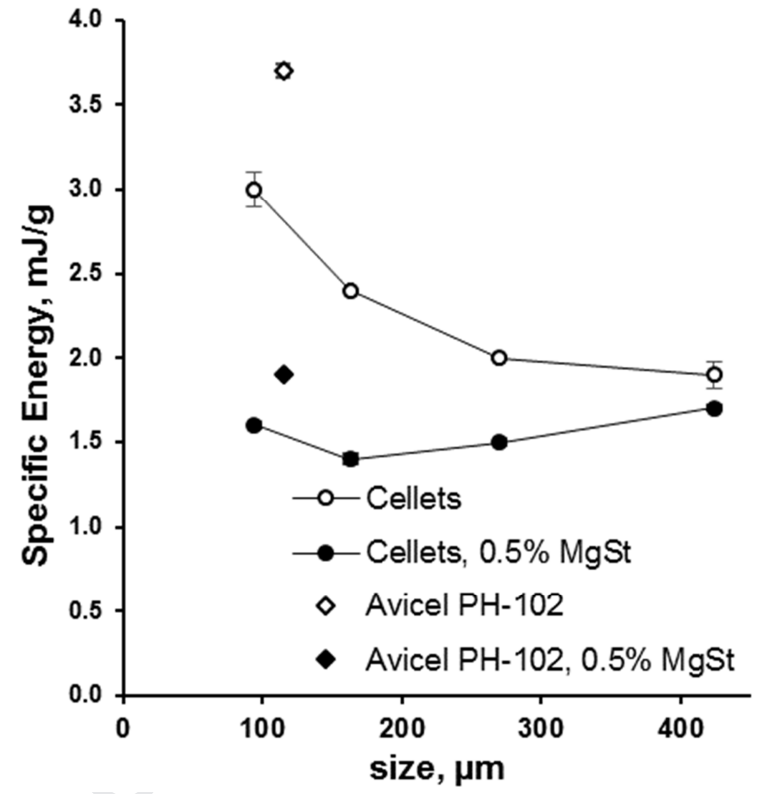

534 Figure 9. Dynamic angle of repose at different rotation speed (A) and Specific Energy (B) for 535 different Cellets' particle size and Avicel PH-102 without and with MgSt.

537 Table 3. Dynamic properties of Cellets measured with FT4 powder rheometer

\begin{tabular}{|c|c|c|c|c|c|c|c|c|}
\hline & \multicolumn{2}{|c|}{$\begin{array}{c}\text { Basic } \\
\text { Flowability } \\
\text { Energy }\end{array}$} & \multicolumn{2}{|c|}{$\begin{array}{l}\text { Specific } \\
\text { Energy }\end{array}$} & \multicolumn{2}{|c|}{$\begin{array}{l}\text { Conditioned } \\
\text { Bulk Density }\end{array}$} & \multicolumn{2}{|c|}{$\begin{array}{c}\text { Aerated } \\
\text { Energy } \\
\text { (at } 40 \mathrm{~mm} / \mathrm{s})\end{array}$} \\
\hline & Av & SD & $\mathbf{A v}$ & SD & $\mathbf{A v}$ & SD & Av & SD \\
\hline & $\mathbf{m J}$ & $\mathbf{m J}$ & $\mathrm{mJ} / \mathrm{g}$ & $\mathrm{mJ} / \mathrm{g}$ & $\mathrm{g} / \mathrm{ml}$ & $\mathrm{g} / \mathrm{ml}$ & $\mathbf{m J}$ & $\mathbf{m J}$ \\
\hline Cellets 350 & 106.0 & 2.2 & 1.9 & 0.1 & 0.9 & 0.002 & 76.3 & 0.5 \\
\hline Cellets 200 & 141.0 & 3.5 & 2.0 & 0.0 & 0.8 & 0.003 & 26.0 & 0.6 \\
\hline Cellets 100 & 163.0 & 0.5 & 2.4 & 0.0 & 0.8 & 0.007 & 1.4 & 0.3 \\
\hline Cellets 90 & 187.0 & 0.4 & 3.0 & 0.1 & 0.8 & 0.006 & 0.9 & 0.2 \\
\hline Avicel PH102 & 84.3 & 2.4 & 3.7 & 0.0 & 0.3 & 0.002 & 1.5 & 0.4 \\
\hline Cellets 350, MgSt (0.5\%) & 89.1 & 1.0 & 1.7 & 0.0 & 0.9 & 0.002 & 49.8 & 2.7 \\
\hline Cellets 200, MgSt (0.5\%) & 92.0 & 1.1 & 1.5 & 0.0 & 0.9 & 0.002 & 2.5 & 0.1 \\
\hline Cellets 100, MgSt (0.5\%) & 80.5 & 0.6 & 1.4 & 0.0 & 0.9 & 0.003 & 1.8 & 0.1 \\
\hline Cellets 90, MgSt (0.5\%) & 81.7 & 0.3 & 1.6 & 0.0 & 0.9 & 0.003 & 1.7 & 0.0 \\
\hline Avicel PH102, MgSt (0.5\%) & 57.4 & 0.5 & 1.9 & 0.0 & 0.4 & 0.004 & 0.9 & 0.5 \\
\hline
\end{tabular}



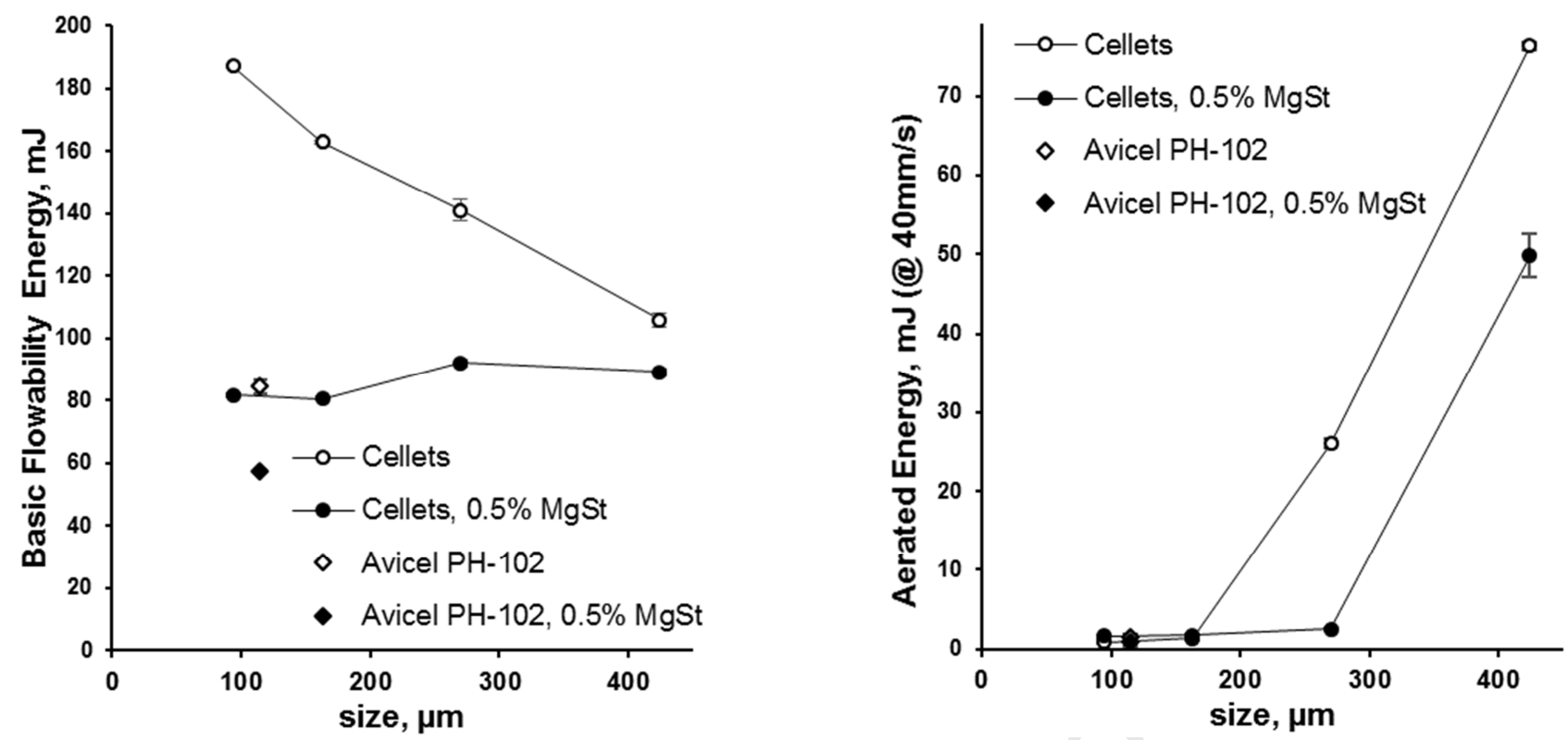

B

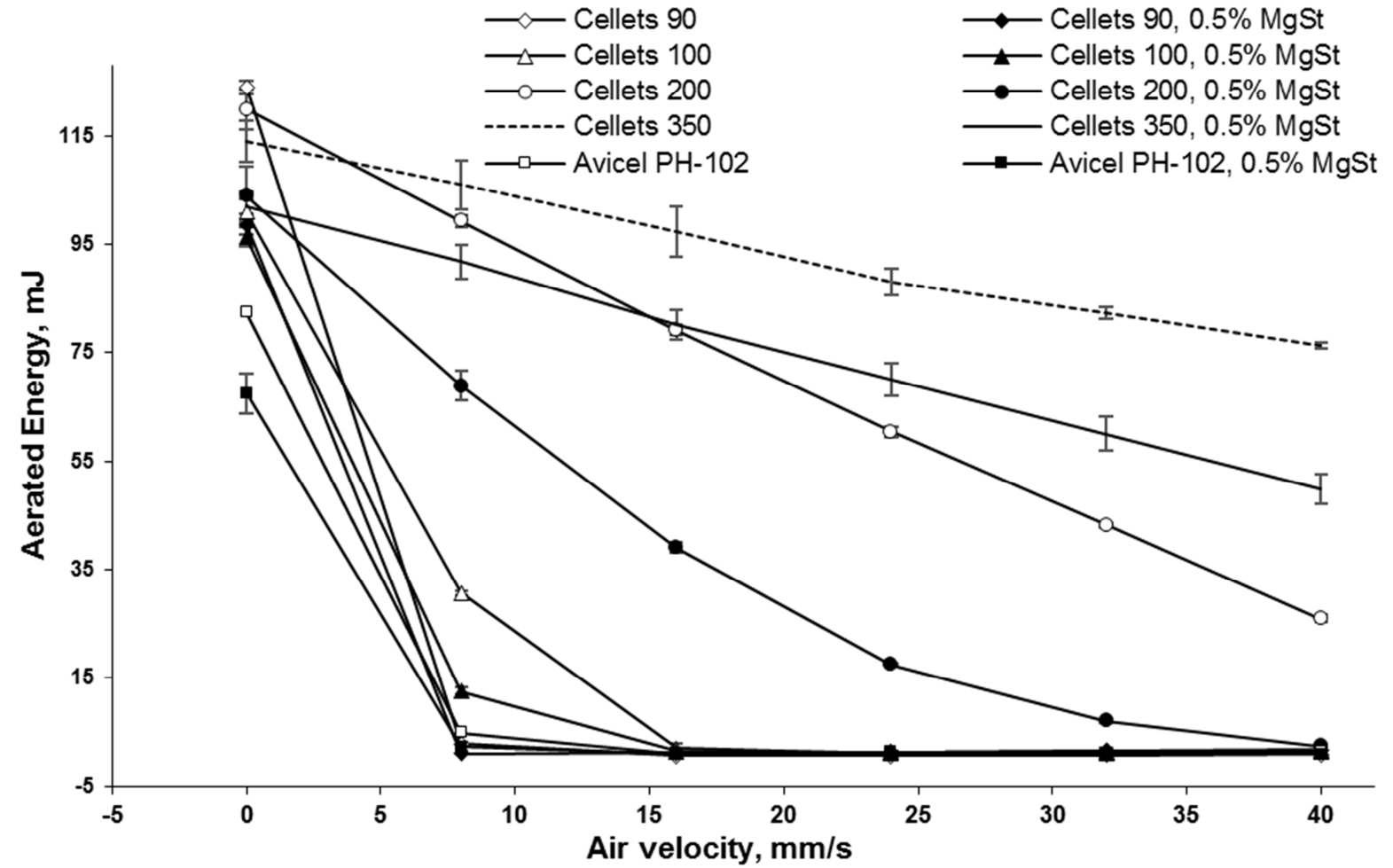

540 Figure 10. Flow Energy measurements of Cellets of varying particle size and Avicel PH-102 541 without and with $0.5 \%$ MgSt. Basic Flowability Energy (A), increasing levels of air velocity 542 on the Aerated Energy (B) and Aerated Energy at $40 \mathrm{~mm} / \mathrm{s}$ air velocity (C). 
544

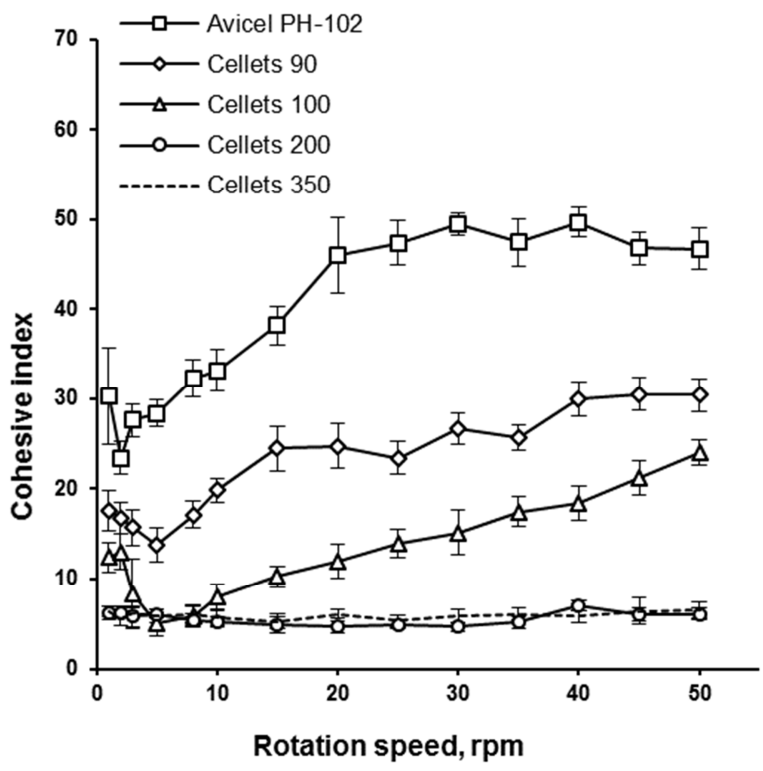

546 Figure 11. Dynamic cohesive index at different rotation speeds on Cellets with varying 547 particle size and Avicel PH-102. 